\title{
Absorption Line Shape of a One-Dimensional Bose Gas
}

\author{
S. K. Yip \\ Institute of Physics, Academia Sinica, Nankang, Taipei 11529, Taiwan
}

\begin{abstract}
We discuss the line shape for an internal state transition for bosonic atoms confined as a one-dimensional gas. Typical line shape is an edge singularity due to the absence of Bose-Einstein Condensation in such systems.
\end{abstract}

PACS numbers: 03.75.Fi

Recent progress in trapped bosonic gases has stimulated a lot of activities in the study of many-body effects in such systems. Most theoretical papers concentrate on effects due to the existence of the Bose-Einstein Condensate (BEC) and the associated macroscopic wave-function. With current techniques, it is likely that one can as well study an effectively one-dimensional (1D) trapped gas [1]. This situation occurs when the bosons are put in a trap which is tightly confining in two directions ( $y$ and $z$ ) but is essentially free in the third $(x)$, and with particles occupying only the lowest quantized subband for motions in the $y-z$ plane. This system is unique in that, for any finite interaction among the bosons, there is no Bose-Einstein Condensation even at zero temperature. [2]. The long-wavelength quantum fluctuations destroy the phase coherence of the system in the macroscopic limit. For a given density $n$, the occupation number $N_{0}$ of the lowest single particle state, though in general increases with the size and hence the total number of particles $N$ in the system, is nevertheless only a negligible fraction of the total $\left(\lim _{N \rightarrow \infty} \frac{N_{0}}{N} \rightarrow 0\right)$. Though many properties of 1D bose gas have been studied in the past [2], the subject remains somewhat academic since there exists no physical realization. The possibility of realizing this 1D bose gas has already stimulated some more theoretical work on this system recently [3, 4 ,

The present 1D bosonic system is closely related to its fermionic counterpart; and they are collectively known as the "Luttinger liquids" [5]. One-dimensional fermionic systems have been studied much more extensively in the context of quantum wires, carbon nanotubes [6,7] etc. There the one-dimensional nature leads to the absence of fermi liquid behavior, so the properties of the system is qualitatively different from its non-interacting counterpart.

The atomically trapped 1D bose gas offers us an excellent new opportunity to study the peculiar properties of quantum systems in reduced dimensions, in particular the consequence due to the absence of BEC. Not only that this is the first 1D bosonic system available, it provides advantages over the mentioned 1D fermionic cases in that the present system is intrinsically clean, and moreover numerous atomic and optical tools can now be used. It may even be possible to further study novel systems such as mixtures etc.

In this paper we consider one such example, namely the absorption line shape of an optical transition in this 1D bose gas. We imagine initially a quasi-1d system of bosons in their internal ground state (referred to as the 'a-atoms' below). An incident optical wave excites one of the atoms to a different internal state. We are interested in the absorption 
line shape of this process; i.e., the probability that the absorption takes place at a given frequency $\Omega$. We shall consider the problem at $T=0$, paying particular attention to the special feature due to the absence of BEC. An analogous experiment has already been done in a three dimensional trap for the $1 \mathrm{~s} \rightarrow 2 \mathrm{~s}$ transition in hydrogen [8]. For a uniform 3D bose gas at $T=0$, the line is expected to be narrow, Lorentzian (see, e.g. [9], c.f. however [8]) with a width governed by the small 'gaseous parameter(s)' $\left(n_{3 d} a^{3}\right)^{1 / 2}$. Here $n_{3 d}$ is the particle number density in $3 \mathrm{~d}$ and $a$ ('s) is (are) the s-wave scattering length(s) for scattering among the bosons. For this three dimensional case, the main weight of the transition comes from exciting a particle from momentum $\vec{p}=0$. There is a macroscopic number of such particles. For a weakly interacting gas, the potential felt by the rest of the bosons is only slightly modified after the transition. We shall see that the situation in $1 \mathrm{D}$ is very different due to the absence of BEC. Exciting a single particle necessitates a substantial rearrangement of the relative motion among the rest of the particles, i.e., emission of a large number of phonons. The basic line shape is typically an edge singularity.

We shall then consider an atomic trap with tight confinement in the $y$ - $z$ directions. For definiteness we shall assume that the confinement potentials in these directions are isotropic and harmonic, with frequency $\omega_{\perp}$. We shall consider the case where the atoms are limited within $0<x<L$ but otherwise free. A weak harmonic trap potential along the $\mathrm{x}$-axis has been considered in the past [10,11], and the low dimensional effects have been shown to be significantly reduced. We shall assume that such a potential is absent here. We shall further ignore the effect due to finiteness of $L$, a condition which we shall return below.

The bosonic system is described by a Hamiltonian containing the kinetic energy and a short-range interaction among the bosons. Anticipating that we shall eventually study the system in the quasi-1d limit, the field operator for the original bosons, referred to hereafter as the a-bosons, is expanded as $\psi_{a}(\vec{r})=\psi_{a}(x) \chi_{0}\left(\vec{r}_{\perp}\right)+\sum_{j} \psi_{a j}(x) \chi_{j}\left(\vec{r}_{\perp}\right)$ where $\psi_{a}(x)$ is the annihilation operator for the a-bosons in the lowest subband (0) where the transverse wavefunction is $\chi_{0}\left(\vec{r}_{\perp}\right)$. $\psi_{a j}(x)$ 's $(j=1,2, \ldots)$ are the corresponding operators for the higher subbands (wavefunctions $\chi_{j}\left(\vec{r}_{\perp}\right)$ ). In the ground state $\left|G>, \psi_{a j}(x)\right| G>=0$ since all particles reside in the lowest subband. We shall see that $\psi_{a j}$ for $j=1,2, \ldots$ will not appear anywhere below, and can pretend that the expansion of $\psi_{a}(\vec{r})$ will consist thus only the term involving the lowest subband. The extent of the wavefunction $\chi_{0}$ in the $\mathrm{y}-\mathrm{z}$ direction will be denoted as $a_{\perp}$. For harmonic trapping potentials in these directions $a_{\perp}=\sqrt{\frac{\hbar}{m \omega_{\perp}}}$.

We shall not write down the interaction part of the Hamiltonian involving the a-bosons explicitly since we won't need it below. For delta-function type interactions it is possible to write down all the formal many-body wavefunctions [12]. Unfortunately manipulations of such wavefunctions are typically very mathematically involved. Moreover we shall eventually be interested in 'final' states where an a-atom has been excited internally. Such wavefunctions, where effectively there is a 'foreign' atom present, are not known (with an exception noted below). We thus proceed rather differently and follow Haldane [5], concentrating only on the low energy excitations which are density oscillations in the system. The field operator $\psi_{a}(x)\left(=\sum_{p} a_{p} e^{i p x} / \sqrt{L}\right)$ describing the motion along $x$ is re-expressed in terms of density $n(x)$ and phase $\phi(x)$ by $\psi_{a}(x)=[n(x)]^{1 / 2} e^{i \phi(x)}$. The effective Hamiltonian for the $1 \mathrm{~d}$ motion can be written as

$$
H_{0}=\frac{\hbar}{2 \pi} \int d x\left[v K(\nabla \phi)^{2}+\frac{v}{K}(\nabla \theta)^{2}\right]
$$


where $\nabla \theta$ is related to the number density fluctuations $\delta n(x)$ by $\nabla \theta=\pi \delta n$. $\phi(x)$ and $\theta(x)$ must be considered as operators satisfying the commutation relation $\left[\phi(x), \nabla_{x^{\prime}} \theta\left(x^{\prime}\right)\right]=$ $i \pi \delta\left(x-x^{\prime}\right) . \quad v$ is the density (sound) velocity wave of the system, and $K$ is the (dimensionless) Luttinger liquid interaction parameter. $v K=\pi n_{o} \hbar / m$ and $K$ is related to the compressibility of the system via $K=\pi v \hbar\left(\partial n_{o} / \partial \mu\right)$ where $n_{o}$ is the linear number density and $\mu$ is the chemical potential of the system. $K$ depends on the interaction among the bosons. For short range interactions, $K$ in principle have already been obtained [5, 12] but there is no general analytic form known. We simply note here that $K=1$ for the strongly repulsive (impenetrable) limit while $K \rightarrow \infty$ if the interaction among the bosons is weak.

$H_{0}$ can be diagonalized easily [5] since it is quadratic. It is useful to introduce bosonic operators $b_{q}$ and $b_{q}^{\dagger}$ which describe the sound modes of the system:

$$
\begin{aligned}
& \theta(x)=-i \sum_{q \neq 0}\left|\frac{\pi K}{2 q L}\right|^{1 / 2} \operatorname{sgn}(q) e^{i q x}\left(b_{-q}^{\dagger}+b_{q}\right) \\
& \phi(x)=-i \sum_{q \neq 0}\left|\frac{\pi}{2 q L K}\right|^{1 / 2} e^{i q x}\left(b_{-q}^{\dagger}-b_{q}\right)
\end{aligned}
$$

Then, apart from a constant, $H_{0}=\sum_{q \neq 0} \hbar \omega_{q} b_{q}^{\dagger} b_{q}$ where the mode frequencies are $\omega_{q}=v|q|$.

Now we consider the external optical field responsible for the transition in internal state. Since the atom with a new internal state is distinguishable from the original atoms, we shall call the resulting atom the 'c-atom'. For definiteness we consider only the 'Doppler-free' part of the spectrum ( no momentum transfer from the exciting laser beam(s)) and assume that the perturbation responsible for the excitation is uniform throughout the 1D bose gas. The relevant part of the Hamiltonian can be then written as $H_{\mathrm{ex}}=w \int d^{3} \vec{r}\left(\psi_{c}^{\dagger}(\vec{r}) \psi_{a}(\vec{r})+\psi_{a}^{\dagger}(\vec{r}) \psi_{c}(\vec{r})\right)$ where $\psi_{c}(\vec{r})$ is the field operator for the atom in the excited internal state. We are interested in the rate of transition as a function of the excitation frequency $\Omega$. This rate $I(\Omega)$ is given simply by the golden rule:

$$
I(\Omega)=2 \pi \sum_{F}|<F| H_{e x}|G>|^{2} \delta\left(\Omega-\left(E_{F}-E_{G}\right)\right)
$$

where $\mid F>$ are the set of final states. To proceed further we need to know the fate of the c-atom. There are many possibilities and we shall simply consider two extreme limits:

(1) An atom in the internal state $c$ is not affected at all by the trapping potential for the atoms in state a. This is possible if, e.g., this potential is due to a laser with frequency near a dipole resonance for the a-atoms but far away from any of those for $c$. In this case the c-atom escapes from the trap and no longer interacts with the remaining a-atoms.

(2) The c-atom also feels the strong transverse confinement potential. It remains inside the trap and continues to interact with the a-atoms.

We shall treat these two cases in turn.

Case 1: In this case the final state is simply a product state, $|F\rangle=|\Phi>\times| \vec{p}\rangle$, with the $N-1$ a-atoms in state $\mid \Phi>$ (with x momentum $-p_{x}$ ) and the c-atom in the plane wave state $|\vec{p}\rangle$ with momentum $\vec{p}$. The rate for transition into states with given $\vec{p}$ is

$$
I_{\vec{p}}(\Omega)=2 \pi\left|f_{\vec{p}}\right|^{2} \sum_{\Phi}|<\Phi| a_{p x}|G>|^{2} \delta\left(\Omega-\left(E_{\Phi}+\vec{p}^{2} / 2 m+u-E_{G}\right)\right)
$$


where $f_{\vec{p}} \equiv \int d^{2} \vec{r}_{\perp} e^{-i \vec{p}_{\perp} \cdot \vec{r}_{\perp}} \chi_{0}\left(\vec{r}_{\perp}\right)$ is a form factor, and $u$ is the difference in internal energy between the a- and c- atoms. This rate is thus proportional to the spectral function for annihilation of a particle in the 1D bose gas (hereafter $p_{x} \rightarrow p$ )

$$
B\left(p, \omega^{\prime}\right) \equiv \sum_{\Phi}|<\Phi| a_{p}|G>|^{2} \delta\left(\omega^{\prime}-\left(E_{\Phi}-E_{G, N-1}\right)\right)
$$

with $\omega^{\prime}=\Omega+E_{G}-E_{G, N-1}-\vec{p}^{2} / 2 m-u$ and where $E_{G, N-1}$ is the ground state energy of $N-1$ atoms in the $1 \mathrm{D}$ trap. $B\left(p, \omega^{\prime}\right)$ is given by $-\frac{1}{\pi} \operatorname{Im} G_{1}\left(p, \omega^{\prime}\right)$ where

$$
G_{1}(x, t) \equiv i<\psi_{a}^{\dagger}(0,0) \psi_{a}(x, t)>h(t)
$$

The required Green's function can be evaluated by finding the long distance and time

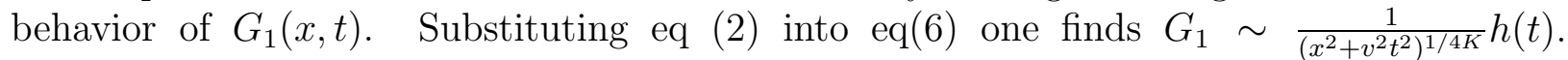
The Fourier transform $G_{1}\left(p, \omega^{\prime}\right)$ and hence $B\left(p, \omega^{\prime}\right)$ for a given $p$ has the scaling form $\sim h\left(\omega^{\prime}-v|p|\right) /\left(\omega^{\prime 2}-v^{2} p^{2}\right)^{1-\alpha}$ with $\alpha=\frac{1}{4 K}$. Since $1<K<\infty$ the line shape is of the form of an edge singularity. For the three dimensional bose gas, the corresponding $B\left(\vec{p}, \omega^{\prime}\right)$ is proportional to a delta function at the Bogoliubov mode frequency (with an additional small incoherent part). The lack of a delta function in the present case reflects the absence of a condensate. If $\vec{p}$ can be independently measured this experiment would be analogous to angle resolved photoemission spectroscopy employed extensively in recent studies of high T-c cuprates [13].

Case 2: In this case the final result depends on the interaction between the a and c atoms, which has the general form $g_{a c} \int d \vec{r} \psi_{c}^{\dagger}(\vec{r}) \psi_{c}(\vec{r}) \psi_{a}^{\dagger}(\vec{r}) \psi_{a}(\vec{r})$. Here $g_{a c}$ is defined in 3D and is related to the 3D scattering length $a_{a c}$ by $g_{a c}=4 \pi \hbar a_{a c} / m$. We express $\psi_{c}(\vec{r})$ also in subbands $\psi_{c}(\vec{r})=\sum_{j} \psi_{c j}(x) \chi_{c j}\left(\vec{r}_{\perp}\right)$. The final states in eq (3) in general can involve terms that are off-diagonal in the band index $j$ for the c-atom due to the interaction between a and c. Such processes correspond to the possibility that the transverse motion of the catom be modified due to the interaction with the a-atoms. For a tight trap however, these contributions are small in the dilute limit. They involve the parameter $n_{o} g_{a c} / \pi a_{\perp}^{2} \omega_{\perp} \sim$ $a_{a c} / l$. Here $l=1 / n_{o}$ is the average interparticle spacing among the a-atoms. Ignoring thus these contributions, we have $I(\Omega)=\sum_{j}\left|f_{j}\right|^{2} I_{j}(\Omega)$, i.e., the transition line then becomes a superposition of 'lines' involving transition to final states where the c-atom is within a given $j$-th subband, with a weight given by the form factor $f_{j}=\int d \vec{r}_{\perp}\left[\chi_{0}\left(\vec{r}_{\perp}\right) \chi_{c j}^{*}\left(\vec{r}_{\perp}\right)\right]$. The shape of each of these lines is determined by $\operatorname{Im}\left[\mathrm{D}_{j}^{R}(\Omega)\right]$ where

$$
\mathrm{D}_{j}^{R}(t)=-i \int d x_{1} \int d x_{2}<\psi_{a}^{\dagger}\left(x_{1}, t\right) \psi_{c j}\left(x_{1}, t\right) \psi_{c j}^{\dagger}\left(x_{2}\right) \psi_{a}\left(x_{2}\right)>h(t)
$$

which we shall now compute. The effective Hamiltonian is given by the sum of $H_{0}$ involving only the a-atoms and $H_{c j}$ which describes the motion of the c-atom within its $j$-th subband and its interaction with the a-atoms. $H_{0}$ has already been given in eq(目) and the effective $H_{c j}$ is given by $H_{c j}^{(1)}+H_{c j}^{\mathrm{int}}$ where

$$
H_{c j}^{(1)}=\int d x\left\{\frac{\hbar^{2}}{2 m} \frac{\partial \psi_{c j}^{\dagger}(x)}{\partial x} \frac{\partial \psi_{c j}(x)}{\partial x}+\left(\epsilon_{c j}+u\right) \psi_{c j}^{\dagger}(x) \psi_{c j}(x)\right\}
$$

consists of the 'one-body' (kinetic, trap and internal energy) terms and 


$$
H_{c j}^{\mathrm{int}}=g_{a c, j j} \int d x \psi_{c j}^{\dagger}(x) \psi_{c j}(x) \psi_{a}^{\dagger}(x) \psi_{a}(x)
$$

is due to the interaction between the a and c atoms. Here $\epsilon_{c j}$ is the subband energy for the c-atom in its j-th subband and $g_{a c, j j}=g_{a c} \int d^{3} \vec{r}\left|\chi_{c j} \chi_{0}\left(\vec{r}_{\perp}\right)\right|^{2}$ depends on $j$. For small $j$ it is of order $g_{a c} / \pi a_{\perp}^{2}$ if the trap potentials for a and c atoms are similar.

It is convenient to express $\mathrm{D}_{j}^{R}(t)$ in the 'interaction' picture:

$$
\mathrm{D}_{j}^{R}(t)=-i \int d x_{1} \int d x_{2}<\psi_{a}^{\dagger}\left(x_{1}, t\right) \psi_{c j}\left(x_{1}, t\right) \hat{T} e^{-i \int_{0}^{t} H_{c j}^{\mathrm{int}}\left(t^{\prime}\right) d t^{\prime}} \psi_{c j}^{\dagger}\left(x_{2}\right) \psi_{a}\left(x_{2}\right)>h(t)
$$

where $\hat{T}$ is the time-ordering operator and the expectation value is to be calculated with the Hamiltonion $H_{0}+H_{c j}^{(1)}$. One can rewrite eq(10) in momentum space and expand the exponent involving the interaction. $H_{c j}^{\text {int }}$ then produces 'scattering' terms where the momentum of the c-atom is changed from one value to the other. The resulting calculation does not seem to be tractable analytically in general. The problem however can be simplified significantly if we assume that $g_{a c, j j}$ is small (condition given below) and concentrate again on the low frequency limit, i.e., just above the threshold for transition into the $j$-th subband (given by $\xi_{c j}=\epsilon_{c j}-\epsilon_{0}-\mu+u+g_{a c, j j} n_{1 d}$ to lowest order in $\left.g_{a c}\right)$. In this case transitions must be made to states where the momenta $p$ of the c-atom are small. Provided that $p<<m v$, then the kinetic energy (of motion along $\mathrm{x}$ ) transferred to the c-atom $p^{2} / 2 m$ is much less than that to the density oscillations of a-atoms $v p$. Thus so long as we are interested in frequency deviations $\Delta \Omega$ from the threshold which satisfy $\Delta \Omega<<m v^{2}$, one can ignore the kinetic energy of the motion of the c-atoms along $x$. For an impenetrable bose gas this amounts to limiting ourselves to $\Delta \Omega<<\pi^{2} \hbar^{2} / m l^{2}$. Ignoring thus the first term in $H_{c j}^{(1)}$ and rewriting the result back in real space, we obtain

$$
\mathrm{D}_{j}^{R}(t)=-i L e^{-i \xi_{c j} t}<\psi_{a}^{\dagger}(0, t) \hat{T} e^{-i \int_{0}^{t} \tilde{H}_{a, i n t}\left(t^{\prime}\right) d t^{\prime}} \psi_{a}(0)>h(t)
$$

where the effective interaction Hamiltonian $\tilde{H}_{a, i n t}$ acts only on the a-atoms and is given by $g_{a c, j j} \psi_{a}^{\dagger}(0) \psi_{a}(0)$. This result can be understood physically as follows: the external optical field produces an a $\rightarrow \mathrm{cj}$ transition at $t=0$ and at a general location say $x_{0}$, annihilating an a-atom while producing a c-atom there in the $\mathrm{j}$-th subband. Since the $c$ atom moves with velocity $p / m$ whereas the sound waves move with the speed $v$, at small $p$ the c-atom is essentially stationary. For $t>0$, due to the c-atom created, a delta-function interaction at $x_{0}$ acts on the a-atoms. We are interested in the overlap between the initial state with an a-atom destroyed at $x_{0}$ and the final states with this extra interaction potential. This overlap is independent of the location of the transition $x_{0}$ which then has been set to 0 . The problem has thus become similar to that of X-ray absorption in solid state physics. There initially $(t<0)$ one has an electron gas in its ground state. At $t=0$ an X-ray photon is absorbed which creates a charged nuclei with an extra electron added to the electron gas, which at $t>0$ feels an extra local potential due to the charged nuclei. Notice however there are slight differences. Here for $t<0$ we have an equilibrium 1D interacting bose gas and at $t>0$ there is one less boson than initially.

The correlation function in (11) and thus the line shape can be found analogous to the X-ray problem [14]. The expectation value needed can be rewritten as $\left\langle\psi^{\dagger}(0) e^{-i \tilde{H} t} \psi(0)\right\rangle$ where $\tilde{H}=H_{0}+\tilde{H}_{\text {int }}$. $H_{0}=\sum \hbar \omega_{q} b_{q}^{\dagger} b_{q}$ and $\tilde{H}_{\text {int }}=g_{a c, j j} \delta n(0)$ can both be written in 
terms of $b_{q}$ and $b_{q}^{\dagger}$. The annihilation operator $\psi \sim e^{i \phi}$ acts like a displacement operator for these bosons: $e^{i \phi} b_{q} e^{-i \phi}=b_{q}-\left|\frac{\pi}{2 q L K}\right|^{1 / 2}$. Thus the expectation value is the same as $<\hat{T} e^{-i \int_{0}^{t}\left(g_{a c, j j}-\pi v_{N}\right) \delta n\left(0, t^{\prime}\right) d t^{\prime}}>$ calculated with the Hamiltonian $H_{0}$. Here we have defined $v_{N} \equiv v / K$. The calculation can be easily done since $H_{0}$ is quadratic in the boson operators $b_{q}$ and $b_{q}^{\dagger}$. The long time dependence is given by, apart from a part oscillating sinusoidally with $t$ which contributes to the line shift, $\mathrm{D}_{j}^{R}(t) \sim 1 / t^{\alpha_{j}^{\prime}}$ and thus the line shape $\sim 1 /[\Delta \Omega]^{1-\alpha_{j}^{\prime}}$ with $\alpha_{j}^{\prime}=\frac{1}{2 K}\left[1-\frac{g_{a c, j j}}{\pi v_{N} \hbar}\right]^{2}$. Notice that the line shape is in general different for transition to different subbands of the 'c-atom'.

It is instructive to compare this result with that of the X-ray problem when the interaction between the nucleus after the X-ray and the electrons is modelled by a delta-function interaction of strength $V$. There the long time behavior of the corresponding correlation function is given by $1 / t^{\alpha_{X}}$ and the line shape is $\sim 1 /[\Delta \Omega]^{1-\alpha_{X}}$ with $\alpha_{X}=[1+N(0) V]^{2}$, where $N(0)$ is the density of states at the fermi level for the electron gas [14]. Here, the interaction strength is replaced by $g_{a c, j j}$ and the density of states is replaced by $\frac{\partial n}{\partial \mu}=\frac{1}{\pi v_{N} \hbar}$. In both cases the power law line shapes (for $\alpha_{X} \neq 0$ and $\alpha_{j}^{\prime} \neq 0$ ) are due to 'orthogonal catastrophe', that the overlap between the state just after the excitation and the new ground state of the system vanishes. There are however two differences: the $+\operatorname{sign}$ in $\alpha_{X}$ is replaced by a - sign since now there is one less boson at $t>0$ rather than one more electron in the $\mathrm{X}$-ray absorption problem. The decay of $D_{j}^{R}(t)$ in time is slower ( $\alpha_{j}^{\prime}$ is smaller) if $g_{a c}>0$ : near the location of the excitation, the reduction in local density of the a-atoms is also what an interaction with $g_{a c}>0$ prefers. The factor $1 / 2 \mathrm{~K}$ arises from the fact that we have a one-dimensional interacting bose gas rather than a three-dimensional fermi liquid.

As the repulsive interaction $g_{a c, j j}$ between the a and c atoms increases, $\alpha_{j}^{\prime}$ decreases and the line sharpens up. The above quantitive result for the exponents $\alpha_{j}^{\prime}$ however holds only for small $g_{a c, j j}\left(<<\pi v_{N} \hbar\right)$. In the limit where interactions among all the bosons are strongly repulsive, one reaches the impenetrable limit where the bosons cannot pass each other. In this limit the statistics, i.e., the indistinguishability among the a-bosons and the distinguishability between the a- and c- bosons become irrelevant. One can check that the wavefunctions written down in [15] for a system of identical bosons only can be generalized to the present case if we replace one of the bosons by the foreign c-atom. It can be further verified that the absorption line shape becomes delta functions (one for each subband $\mathrm{cj}$ ) in this impenetrable limit.

The experimental observation of the line shape discussed above should be feasible. The possibility of obtaining a quasi-1D bose gas has already been discussed in ref 四. The above line-shape is applicable so long as $\Delta \Omega_{\sim}^{<\frac{\pi^{2} \hbar}{m l^{2}}} \equiv \omega_{l}$. For hydrogen atoms and with $l \sim 1 \mu m$, $\omega_{l} \sim 100 \mathrm{kHz}$, much larger than the present available experimental resolutions. It is sufficient for the temperature $T$ to be small compared with the line-width. The condition $T<<\omega_{l}$ is satisfied for $T<\mu K$, a temperature readily achievable. A finite length $L$ of the system will break the line into a set of discrete sub-lines with separations of order $\pi^{2} \hbar / m L^{2}$, but the shape of the 'line' can still be observable as long as $L>>l$.

I thank Ite $\mathrm{Yu}$ for a useful correspondence. 


\section{REFERENCES}

[1] M. Olshanii, Phys. Rev. Lett. 82, 4208 (1998); and reference therein

[2] see articles collected in D. C. Mattis, The Many Body Problem, World Scientific, 1993.

[3] A. G. Rojo, J. L. Cohen and P. R. Berman, Phys. Rev. A 60, 1482 (1999)

[4] M. D. Girardeau and E. M. Wright, Phys. Rev. Lett. 84, 5239 (2000)

[5] F. D. M. Haldane, Phys. Rev. Lett. 47, 1840 (1981)

[6] see, e.g., S. Tarucha, T. Honda and T. Saku, Sol State Comm., 94, 413 (1995)

[7] Z. Yao, H. W. Ch. Postma, L. Balents and C. Dekker, Nature, 402, 273 (1999)

[8] T. C. Killian et al, Phys. Rev. Lett. 81, 3807 (1998)

[9] M. Ö. Oktel and L. S. Levitov, Phys. Rev. Lett. 83, 6 (1999)

[10] T. L. Ho and M. Ma, J. Low Temp. Phys. 115, 61 (1999)

[11] D. S. Petrov, G. V. Shlyapnikov and J. T. M. Walraven, Phys. Rev. Lett. 85, 3745 (2000)

[12] E. H. Lieb and W. Liniger, Phys. Rev. 130, 1605 (1963); E. H. Lieb, ibid, 1616.

[13] see, e.g., M. Randeria and J. C. Campuzzano, in Proceedings of the International School of Physics "Erico Fermi", Varenna, 1997 (North Holland)

[14] S. Doniach and E. H. Sondheimer, Green's Functions for Solid State Physicists, Frontiers in Physics, vol 44, Addison-Wesley, 1974.

[15] M. Girardeau, J. Math. Phys. 1, 516 (1960) 